\title{
A counterexample to King's conjecture
}

\author{
Lutz Hille and Markus Perling
}

\begin{abstract}
King's conjecture states that on every smooth complete toric variety $X$ there exists a strongly exceptional collection which generates the bounded derived category of $X$ and which consists of line bundles. We give a counterexample to this conjecture. This example is just the Hirzebruch surface $\mathbb{F}_{2}$ iteratively blown up three times, and we show by explicit computation of cohomology vanishing that there exist no strongly exceptional sequences of length 7 which consist of line bundles.
\end{abstract}

\section{Introduction}

It is a widely open question whether, on a given smooth algebraic variety $X$ (say, complete and smooth), there exists a tilting sheaf. A tilting sheaf is a sheaf $\mathcal{T}$ which generates the bounded derived category $\mathcal{D}^{b}(X)$ of $X$ and $\operatorname{Ext}^{k}(\mathcal{T}, \mathcal{T})=0$ for all $k>0$. For such $\mathcal{T}$, the functor

$$
\mathbf{R H o m}(\mathcal{T}, .): D^{b}(X) \longrightarrow D^{b}(A-\bmod ),
$$

where $A:=\operatorname{End}(\mathcal{T})$ is the endomorphism algebra, induces an equivalence of categories (see [Rud90, Bon90, Bei78]). The existence of a tilting sheaf implies that the Grothendieck group of $X$ is finitely generated and free, so that, in general, such sheaves cannot exist. However, so far there are a number of positive examples known, including projective spaces, del Pezzos, certain homogeneous spaces, and some higher-dimensional Fanos. An obvious testbed for the existence of tilting sheaves are the toric varieties. There is a quite strong conjecture which was first stated by King [Kin97] as follows.

Conjecture 1.1 (King [Kin97]). Let $X$ be a smooth complete toric variety. Then $X$ has a tilting sheaf which is a direct sum of line bundles.

If a tilting sheaf decomposes into a direct sum of line bundles, its direct summands $\mathcal{T}=\bigoplus_{i=1}^{t} \mathcal{L}_{i}$ form a so-called strongly exceptional sequence, i.e. $\operatorname{Ext}^{k}\left(\mathcal{L}_{i}, \mathcal{L}_{j}\right)=0$ for all $i, j$ and all $k>0$, and (after possibly reordering the $\left.\mathcal{L}_{i}\right) \operatorname{Hom}\left(\mathcal{L}_{i}, \mathcal{L}_{j}\right)=0$ for $i>j$. Moreover, $t$ is the rank of the Grothendieck group of $X$.

It would be very nice if easily computable tilting sheaves on toric varieties existed, and indeed many positive examples in favor of the conjecture are known (see [CM04, Kaw05, Hil04], and also [AH99, AKO04, BP05, CS06] for related results). Computer experiments also look promising in many directions. However, the conjecture has remained somewhat mysterious so far and, as it turns out, is false in general. It is the purpose of this paper to present a counterexample.

Our counterexample is the toric surface $X$ as shown in Figure 1, which can be obtained by iteratively blowing up the Hirzebruch surface $\mathbb{F}_{2}$ three times. In coordinates, the primitive vectors of its rays are given as shown in Figure 1.

Received 28 February 2006, accepted in final form 6 April 2006.

2000 Mathematics Subject Classification 14M25, 18E30 (primary), 14J81 (secondary).

Keywords: toric varieties, derived categories, exceptional sequences.

The second author is supported by a grant of the German Research Council (DFG).

This journal is (c) Foundation Compositio Mathematica 2006. 


\section{Hille And M. Perling}

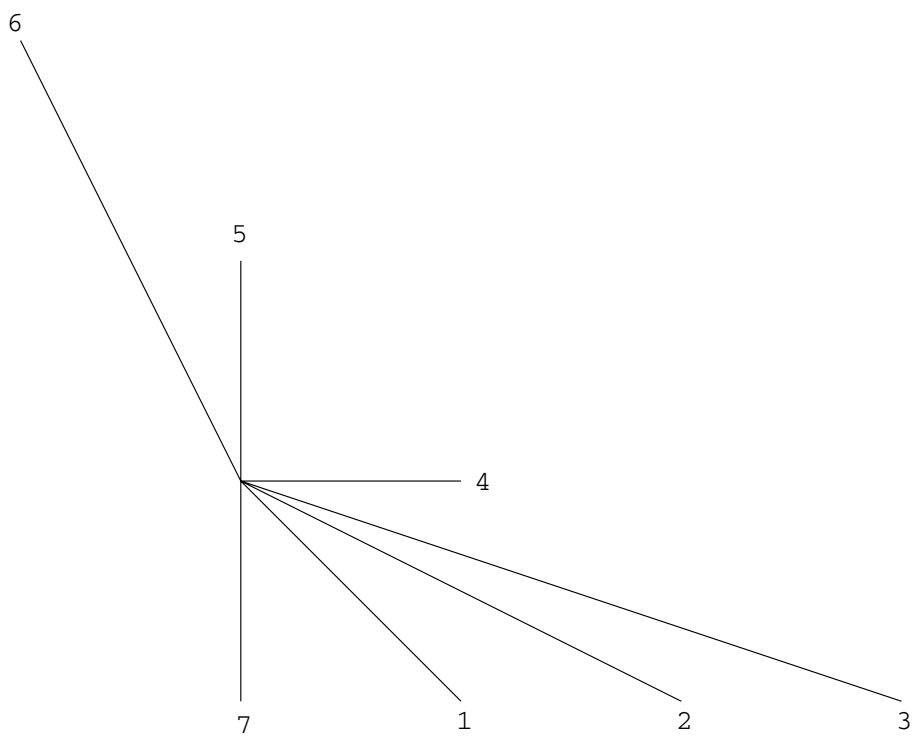

Figure 1. The fan. The endpoints of the lines indicate the position of the primitive vectors: $l_{1}=$ $(1,-1), l_{2}=(2,-1), l_{3}=(3,-1), l_{4}=(1,0), l_{5}=(0,1), l_{6}=(-1,2), l_{7}=(0,-1)$.

Note that the rank of the Grothendieck group of $X$ is 7 . To show that no strongly exceptional sequences of length 7 exist on this surface, we will perform explicit computations in the Picard group to determine cohomology vanishing. More precisely, note that if $\mathcal{L}_{1}, \ldots, \mathcal{L}_{t}$ is a strongly exceptional sequence, then also $\mathcal{L}_{1} \otimes \mathcal{L}^{\prime}, \ldots, \mathcal{L}_{t} \otimes \mathcal{L}^{\prime}$ is strongly exact, where $\mathcal{L}^{\prime}$ is any line bundle. So one can assume without loss of generality that the sequence contains the structure sheaf. Then a necessary condition for the bundles in the sequence is that all of the higher cohomology groups of the bundles and of their dual bundles vanish, i.e. $H^{k}\left(X, \mathcal{L}_{i}\right)=H^{k}\left(X, \mathcal{L}_{i}^{*}\right)=0$ for all $i$ and all $k>0$. This is a rather strong condition, and our main computation will be to compile a complete list of such bundles for our surface $X$. After having obtained this classification, we deduce by simple inspection that no strongly exceptional sequence of length 7 and consisting of line bundles exists.

\section{Overview}

In $\S 2$ we state everything we need to know about cohomology of line bundles on toric surfaces and we describe in more detail our method of computation. In $\S 3$ all bundles are classified which have the property that the higher cohomologies of the bundles themselves and of their dual bundles vanish. In $\S 4$ we show by inspection that no strongly exceptional sequences of length 7 exist on $X$.

\section{The setup}

In this section we recall basic facts on cohomology of line bundles on a toric surface and we describe our method of computation. For general information about toric varieties we refer to [Oda88, Ful93].

\subsection{Generalities on toric line bundles}

Let $X$ be a complete smooth toric surface on which the torus $T$ acts. The variety $X$ is described by a fan $\Delta$ which is contained in a two-dimensional vector space $N_{\mathbb{R}}:=N \otimes_{\mathbb{Z}} \mathbb{R}$, where $N \cong \mathbb{Z}^{2}$ is the group of one-parameter subgroups of $T$. We denote by $\Delta(1)$ the set of rays, that is, of onedimensional cones of $\Delta$. As $X$ is a complete surface, the fan is completely determined by the rays. We denote the primitive vectors of the rays by $l_{1}, \ldots, l_{n}$, enumerated in counterclockwise order. 


\section{A COUnTEREXAmple to King's CONJECture}

To any $l_{i}$ there is associated a $T$-invariant divisor $D_{i}$, and every divisor $D$ can, up to linear equivalence, written as a sum of these invariant divisors, i.e. $D=\sum_{i=1}^{n} c_{i} D_{i}$. We denote by $M \cong \mathbb{Z}^{2}$ the character group of the torus acting on $X$ and we set $M_{\mathbb{R}}:=M \otimes_{\mathbb{Z}} \mathbb{R}$. The lattice $N$ is in a natural way dual to $M$, and the primitive vectors $l_{i}$ are integral linear forms on $M$ (and on $M_{\mathbb{R}}$, respectively). There is a short exact sequence

$$
0 \longrightarrow M \stackrel{A}{\longrightarrow} \mathbb{Z}^{\Delta(1)} \longrightarrow \operatorname{Pic}(X) \longrightarrow 0
$$

where the matrix $A$ is composed of the $l_{i}$ as row vectors. This sequence is split exact. More precisely, if we choose two of the $l_{i}$, for instance $l_{n-1}$ and $l_{n}$, which form a $\mathbb{Z}$-basis of $N$, then the divisors $D_{1}, \ldots, D_{n-2}$ form a $\mathbb{Z}$-basis of $\operatorname{Pic}(X)$. So every divisor $D$ has a unique representation $D=\sum_{i=1}^{n-2} c_{i} D_{i}$.

Now let $D=\sum_{i=1}^{n} c_{i} D_{i}$ be any $T$-invariant divisor. In a natural way, $D$ defines an affine hyperplane arrangement $\mathcal{H}_{D}=\left\{H_{1}, \ldots, H_{n}\right\}$ in the vector space $M_{\mathbb{R}}$, where

$$
H_{i}=\left\{m \in M_{\mathbb{R}} \mid l_{i}(m)=-c_{i}\right\} .
$$

All information on the cohomology of the line bundle $\mathcal{O}(D)$ is contained in the chamber structure $\mathcal{H}_{D}$ (or, more precisely, in the intersection of this chamber structure with the lattice $M$ ). Recall that the $T$-action induces an eigenspace decomposition on the cohomology groups of $\mathcal{O}(D)$ :

$$
H^{k}(X, \mathcal{O}(D))=\bigoplus_{m \in M} H^{k}(X, \mathcal{O}(D))_{m}
$$

for all $k \geqslant 0$. The dimension of $H^{k}(X, \mathcal{O}(D))_{m}$ as a vector space is determined by the signature of $m$ with respect to the arrangement $\mathcal{H}_{D}$.

Definition 2.1. Let $D=\sum_{i=1}^{n} c_{i} D_{i}$ be a $T$-invariant divisor on $X$. Then, for every $i=1, \ldots, n$, we define a signature

$$
\Sigma_{i}^{D}: M \longrightarrow\{+,-, 0\}
$$

where $\Sigma_{i}^{D}(m)=+$ if $l_{i}(m)>-c_{i}, \Sigma_{i}^{D}(m)=-$ if $l_{i}(m)<-c_{i}$ and $\Sigma_{i}^{D}(m)=0$ if $l_{i}(m)=-c_{i}$. Moreover, we denote

$$
\Sigma^{D}: M \longrightarrow\{+,-, 0\}^{n},
$$

where $\Sigma^{D}(m)$ is the tuple $\left(\Sigma_{1}^{D}(m), \ldots, \Sigma_{n}^{D}(m)\right)$.

In the following, we mostly work with only one $D$ at a time, whose values for the $c_{i}$ will be clear from the context. So we usually omit the reference to $D$ in the notation, i.e. we mostly write $\Sigma(m)$ instead of $\Sigma^{D}(m)$.

Given the signature $\Sigma^{D}(m)$, the computation of $H^{k}(X, \mathcal{O}(D))_{m}$ is straightforward. We use a method which for toric surfaces can easily be derived from the standard textbook treatment for cohomology computation for line bundles (we refer to [EMS00, HKP05] for an explicit description for general toric varieties, see also [AH99, Lemma 3.4]).

For a given signature $\Sigma^{D}(m)$, a --interval is a connected sequence of - with respect to the circular order of the $\rho_{i}$. For example, assume that $\Delta(1)$ consists of seven elements enumerated in circular order. Then the signature +--++-+ has two - -intervals. Note that due to the circular ordering of the rays, the signature --+++-- has only one - -interval.

Lemma 2.2. Let $D$ be a $T$-invariant divisor on a toric surface $X$ and $m \in M$. Then we have

$$
\operatorname{dim} H^{1}(X, \mathcal{O}(D))_{m}= \begin{cases}\text { the number of --intervals }-1 & \text { if there exists at least one --interval, } \\ 0 & \text { otherwise. }\end{cases}
$$




\section{Hille And M. Perling}

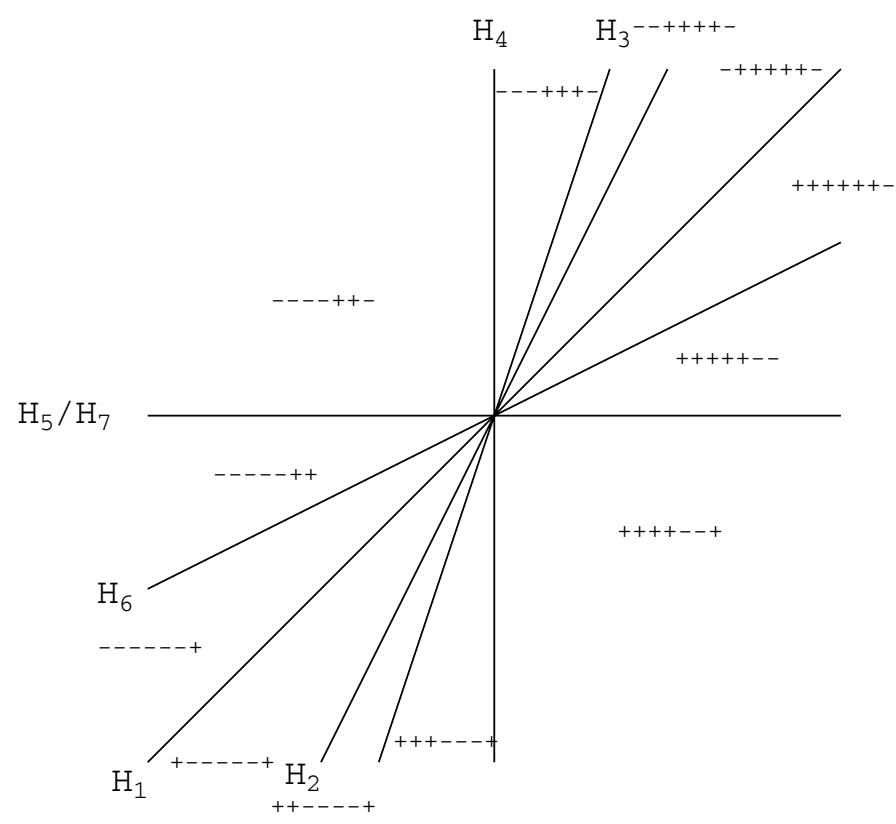

Figure 2. The central arrangement.

and

$$
\operatorname{dim} H^{2}(X, \mathcal{O}(D))_{m}= \begin{cases}1 & \text { if } \Sigma^{D}(m)=\{-\}^{n} \\ 0 & \text { otherwise }\end{cases}
$$

\subsection{Method of computation}

Let $\mathcal{L}_{1}, \ldots, \mathcal{L}_{t}$ be a strongly exceptional sequence of line bundles, i.e. we have $\operatorname{Ext}^{k}\left(\mathcal{L}_{i}, \mathcal{L}_{j}\right)=0$ for all $i, j$ and all $k>0$. There is a natural isomorphism $\operatorname{Ext}^{k}\left(\mathcal{L}_{i}, \mathcal{L}_{j}\right) \cong H^{k}\left(X, \mathcal{L}_{i}^{*} \otimes \mathcal{L}_{j}\right)$, where $\mathcal{L}_{i}^{*}=\operatorname{Hom}\left(\mathcal{L}_{i}, \mathcal{O}_{X}\right)$ denotes the dual bundle. By this we can assume without loss of generality that one of the $\mathcal{L}_{i}$ is just the structure sheaf $\mathcal{O}_{X}$, i.e. $\mathcal{L}_{1}, \ldots, \mathcal{L}_{t}$ is a strongly exceptional sequence if and only if $\mathcal{L}_{i}^{*} \otimes \mathcal{L}_{1}, \ldots, \mathcal{L}_{i}^{*} \otimes \mathcal{L}_{t}$ is a strongly exceptional sequence. If $\mathcal{O}_{X}$ is part of the sequence, this in turn implies a rather strong condition on the cohomologies of the other bundles. Namely, for every $\mathcal{L}_{i}$ we have

$$
H^{k}\left(X, \mathcal{L}_{i}\right)=H^{k}\left(X, \mathcal{L}_{i}^{*}\right)=0 \text { for all } k>0
$$

Thus, to show that our toric surface does not have a strongly exceptional sequence of length 7 , we proceed in two steps as follows.

(i) We classify all line bundles where higher cohomologies of the bundle itself as well as of its dual vanish. It turns out that the list of such bundles has a rather short description, although it is not finite.

(ii) After having obtained the list, we show by exclusion that there are no strongly exceptional sequences of length 7 .

Example 1. Figure 2 shows the arrangement that belongs to the structure sheaf. We see that this arrangement is central and induces a chamber decomposition of the space $M_{\mathbb{R}}$, consisting of unbounded chambers. To every chamber there is associated a signature which we have indicated in the picture. Note that, in fact, there are some more signatures which are not shown. For instance, the points lying on the line between the chambers with signatures ++++++- and -+++++have signature $0+++++-$. The origin has signature 0000000 . 
A Counterexample to King'S COnjecture

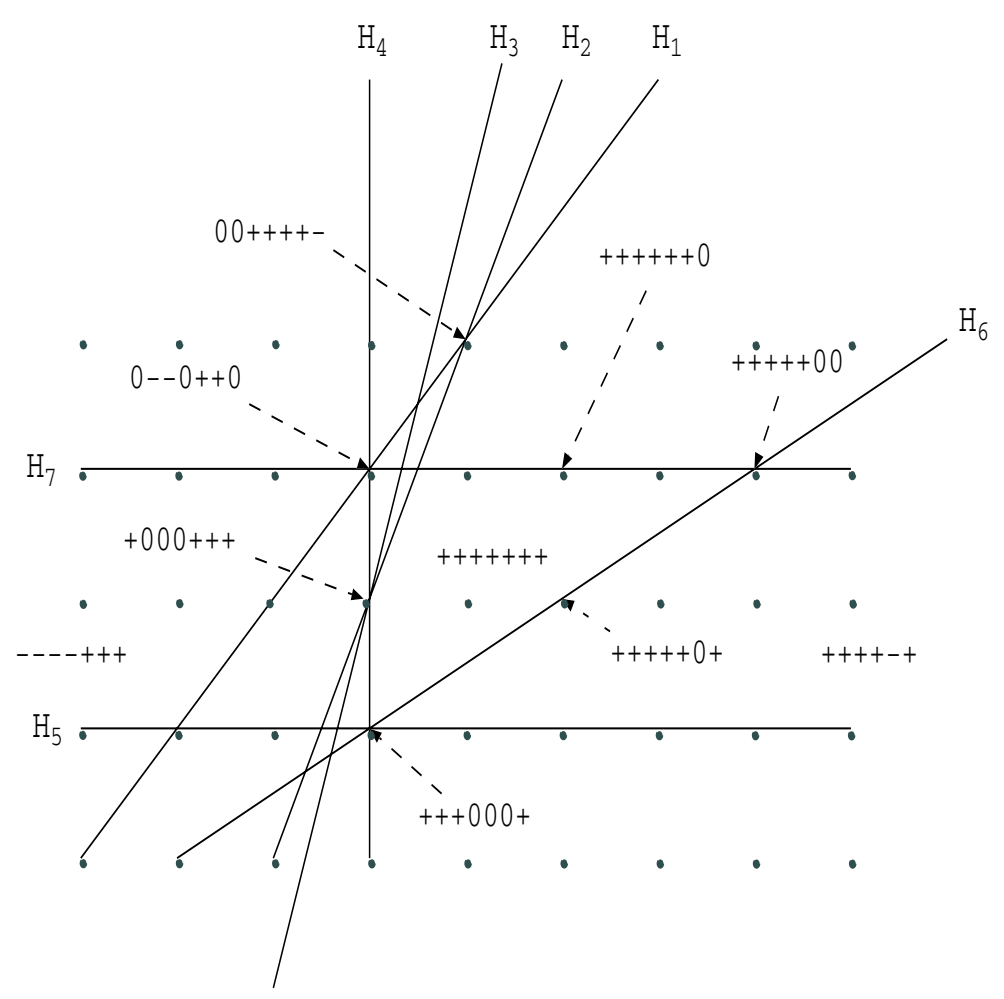

Figure 3. A deformation of the central arrangement.

Figure 3 shows a deformation of this central arrangement that belongs to the divisor $D=$ $-\left(4 D_{1}+7 D_{2}+11 D_{3}+4 D_{4}+2 D_{5}\right)$. As we can see, moving the hyperplanes creates new chambers with new signatures. There are two new unbounded chambers with signatures ----+++ and +++++-+ , respectively, which obviously have no influence on the cohomology of $\mathcal{O}(D)$. The other chambers are all bounded and thus contain only a finite number of lattice points (i.e. points in $M$ ). We have indicated the signatures of some of these points in the picture. As one can check, most of these signatures do not give rise to nonvanishing cohomology, the only exception being the point with signature +++++++ . Recall that we are interested in the classification of line bundles which have no higher cohomology and whose duals also have no higher cohomology. So, if there is an inequality $l_{i}(m)<-c_{i}$ (or $\left.l_{i}(m)>-c_{i}\right)$, then we have $l_{i}(-m)>c_{i}\left(l_{i}(-m)<c_{i}\right.$, respectively), whereas for $l_{i}(m)=-c_{i}$ we have $l_{i}(-m)=c_{i}$. In our example the signature of $m$ with $\Sigma^{D}(m)=+++++++$ becomes $\Sigma^{-D}(-m)=------$ for the dual bundle, which therefore has nonvanishing $H^{2}$.

We give one more example and some more notation. In many situations it will not be necessary to know the complete signature of some point $m \in M$. Therefore we define the following.

Definition 2.3. A partial signature is given by

$$
\Sigma^{D}: M \longrightarrow\{+,-, 0, *\}^{n}
$$

which is a signature for some subset $I$ of $\{1, \ldots, n\}$ such that $\left(\Sigma^{D}(m)\right)_{i}=\Sigma_{i}^{D}(m)$ for $i \in I$ and $\left(\Sigma^{D}(m)\right)_{i}=*$ for $i \notin I$.

It is convenient for us to use the same symbol for signatures and partial signatures. To exemplify our computations, we prove the following.

LEMMA 2.4. We have $c_{5} \leqslant 5$. 


\section{Hille AND M. Perling}

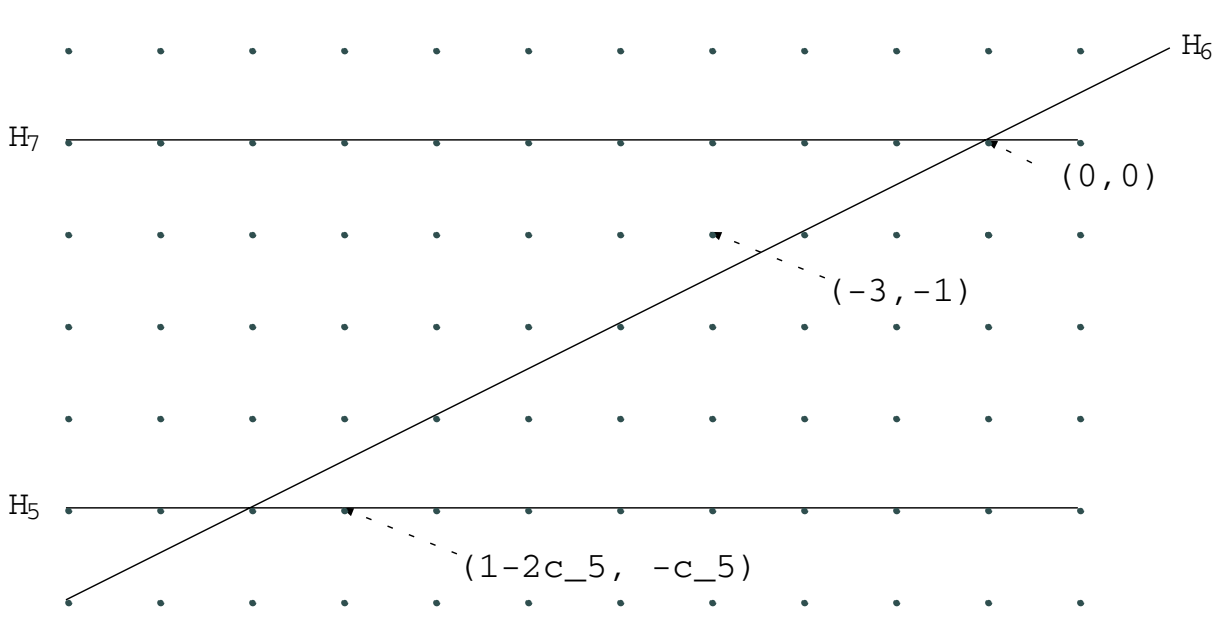

FiguRE 4. A partial arrangement.

Proof. Assume $D=\sum_{i=1}^{5} c_{i} D_{i}$ and $c_{5}>0$. Now consider the point $m$ in $M$ which has the coordinates $\left(1-2 c_{5},-c_{5}\right)$ (see Figure 4$)$.

Its partial signature with respect to the linear forms $l_{5}, l_{6}, l_{7}$ is $\Sigma(m)=* * * * 0-+$. Our aim is to derive conditions on the values of the $c_{i}$. Evidently, any complete signature that is obtained by filling the $*$ has at least one --interval. Moreover, if any of the $*$ becomes a - , the signature has at least two --intervals, and any corresponding line bundle will have nonvanishing $H^{1}$. So, a necessary condition is that $\Sigma_{i}^{D}(m) \in\{+, 0\}$ for $i=1, \ldots, 4$ and any valid divisor $D$. This, in turn, implies that

$$
\begin{aligned}
& c_{1} \geqslant c_{5}-1 \\
& c_{2} \geqslant 3 c_{5}-2 \\
& c_{3} \geqslant 5 c_{5}-3 \\
& c_{4} \geqslant 2 c_{5}-1 .
\end{aligned}
$$

Now the point $(-3,-1)$ has partial signature $\Sigma(-3,-1)=* * * *+++$, and the above conditions on $c_{1}, \ldots, c_{4}$ imply that for $c_{5}>3$ this point always has signature +++++++ , and thus we have nonvanishing $H^{2}$. Hence, we conclude $c_{5} \leqslant 3$.

\section{Classification of line bundles without higher cohomology}

In this section we carry out the complete classification of line bundles for our toric surface which have the property that the higher cohomologies vanish for both the bundle itself and its dual. The result is listed in Table 1 . As explained in the previous section, we can always assume that a line bundle $\mathcal{L}$ is uniquely represented by an invariant divisor $D=\sum_{i=1}^{5} c_{i} D_{i}$, and every tuple of numbers $\left(c_{1}, \ldots, c_{5}\right)$ represents a unique isomorphism class in $\operatorname{Pic}(X)$. As we have already seen in Lemma 2.4, we can assume that $c_{5} \leqslant 3$. Moreover, as it does not matter whether we deal with a bundle or its dual, we can without loss of generality assume $c_{5} \geqslant 0$. So, this leaves us with four possible values for $c_{5}$. Our classification will be done by subsequential case distinctions which on the top level are guided by the four possible values of $c_{5}$.

Note that in the following, for a given bundle we will use phrases such as 'has cohomology' if either the bundle itself or its dual has a nonvanishing higher cohomology group. We prove the following theorem.

Theorem 3.1. Table 1 contains all line bundles on $X$ with no higher cohomology groups. 


\section{A COUnterexample to King'S CONJECture}

TABLE 1. The cohomology free line bundles.

\begin{tabular}{|c|c|c|}
\hline Name & $\left(c_{1}, c_{2}, c_{3}, c_{4}, c_{5}\right)$ & Compatible with \\
\hline$A_{1}$ & $(0,0,1,0,0)$ & $\begin{array}{l}-A_{6}, A_{7},-C_{2}, C_{3},-C_{6}, C_{7},-C_{9}, C_{10} \\
-B_{1, k}, B_{2, k},-B_{3, k}, B_{4, k},-B_{6, k}, B_{7, k}\end{array}$ \\
\hline$A_{2}$ & $(0,1,1,0,0)$ & $\begin{array}{c}-A_{5}, A_{7},-C_{1}, C_{3},-C_{4}, C_{5},-C_{6},-C_{7}, C_{9}, C_{10} \\
-B_{1, k}, B_{3, k},-B_{2, k}, B_{4, k},-B_{5, k}, B_{7, k}\end{array}$ \\
\hline$A_{3}$ & $(0,1,2,1,0)$ & $\begin{array}{c}-A_{4}, A_{7},-C_{1},-C_{3}, C_{4}, C_{5} \\
-B_{2, k}, B_{5, k},-B_{3, k}, B_{6, k},-B_{4, k}, B_{7, k} \text { if } k \geqslant 1\end{array}$ \\
\hline$A_{4}$ & $(1,1,1,0,0)$ & $\begin{array}{c}-A_{3}, A_{7},-C_{1},-C_{2},-C_{3}, C_{7}, C_{9}, C_{10} \\
B_{2, k} \text { if } k \geqslant 2, B_{3, k} \text { if } k \geqslant 2, B_{4, k} \\
-B_{5, k},-B_{6, k}, B_{4, k},-B_{7, k}\end{array}$ \\
\hline$A_{5}$ & $(1,1,2,1,0)$ & $\begin{array}{c}-A_{2}, A_{7},-C_{1}, C_{8}, B_{1, k}, B_{2, k} \text { if } k \geqslant 2,-B_{3, k}, \\
-B_{4, k}, B_{5, k},-B_{6, k}\end{array}$ \\
\hline$A_{6}$ & $(1,2,2,1,0)$ & $\begin{array}{c}-A_{1}, A_{7}, B_{1, k},-B_{2, k}, B_{3, k} \text { if } k \geqslant 2 \\
-B_{4, k}, B_{6, k},-B_{7, k}\end{array}$ \\
\hline$A_{7}$ & $(1,2,3,1,0)$ & $\begin{array}{c}A_{1}, A_{2}, A_{3}, A_{4}, A_{5}, A_{6}, B_{1, k} \text { for } k \geqslant 3, B_{7,1} \\
B_{i, k} \text { for } i=2, \ldots, 7 \text { and } k \geqslant 2 \\
-B_{i, k} \text { for } i=1, \ldots, 7\end{array}$ \\
\hline$B_{1, k}, k \geqslant 2$ & $(k, 2 k-1,3 k-2, k, 1)$ & $\begin{array}{c}-A_{1},-A_{2}, A_{5}, A_{6}, A_{7} \text { if } k \geqslant 3,-A_{7}, \\
B_{1, k-1} \text { if } k \geqslant 2, B_{1, k+1}, B_{2, k-1}, B_{2, k}, B_{3, k-1}, B_{3, k}\end{array}$ \\
\hline$B_{2, k}, k \geqslant 1$ & $(k, 2 k-1,3 k-1, k, 1)$ & $\begin{array}{c}A_{1},-A_{2},-A_{3}, A_{4} \text { if } k \geqslant 2,-A_{6}, A_{7} \text { if } k \geqslant 2,-A_{7}, \\
B_{1, k} \text { if } k \geqslant 2, B_{1, k+1}, B_{2, k-1} \text { if } k \geqslant 2, B_{2, k+1}, \\
B_{4, k-1} \text { if } k \geqslant 2, B_{4, k}, B_{5, k-1} \text { if } k \geqslant 2, B_{5, k}\end{array}$ \\
\hline$B_{3, k}, k \geqslant 1$ & $(k, 2 k, 3 k-1, k, 1)$ & $\begin{array}{c}-A_{1}, A_{2}, A_{4} \text { if } k \geqslant 2,-A_{5}, A_{6} \text { if } k \geqslant 2, A_{7} \text { if } k \geqslant 2,-A_{7}, \\
B_{1, k} \text { if } k \geqslant 2, B_{1, k+1}, B_{3, k-1} \text { if } k \geqslant 2, B_{3, k+1} \\
B_{4, k-1} \text { if } k \geqslant 2, B_{4, k}, B_{6, k-1} \text { if } k \geqslant 2, B_{6, k}\end{array}$ \\
\hline$B_{4, k}, k \geqslant 1$ & $(k, 2 k, 3 k, k, 1)$ & $\begin{array}{c}A_{1}, A_{2},-A_{3}, A_{4} \text { if } k \geqslant 2,-A_{5},-A_{6}, A_{7} \text { if } k \geqslant 2,-A_{7}, \\
B_{2, k}, B_{2, k+1}, B_{3, k}, B_{3, k+1}, B_{4, k-1} \text { if } k \geqslant 2, B_{4, k+1}, \\
B_{7, k-1} \text { if } k \geqslant 2, B_{7, k}\end{array}$ \\
\hline$B_{5, k}, k \geqslant 1$ & $(k, 2 k, 3 k+1, k+1,1)$ & $\begin{array}{c}-A_{2},-A_{4}, A_{5} \text { if } k \geqslant 2, A_{7} \text { if } k \geqslant 2,-A_{7} \\
B_{2, k}, B_{2, k+1}, B_{5, k-1} \text { if } k \geqslant 2, B_{5, k+1} \\
B_{7, k-1} \text { if } k \geqslant 2, B_{7, k}\end{array}$ \\
\hline$B_{6, k}, k \geqslant 1$ & $(k, 2 k+1,3 k+1, k+1,1)$ & $\begin{array}{c}-A_{1}, A_{3},-A_{4},-A_{5}, A_{6} \text { if } k \geqslant 2, A_{7} \text { if } k \geqslant 2,-A_{7}, \\
B_{3, k}, B_{3, k+1}, B_{6, k-1} \text { if } k \geqslant 2, B_{6, k+1}, \\
B_{7, k-1} \text { if } k \geqslant 2, B_{7, k}\end{array}$ \\
\hline$B_{7, k}, k \geqslant 0$ & $(k, 2 k+1,3 k+2, k+1,1)$ & $\begin{array}{c}A_{1} \text { if } k \geqslant 1, A_{2} \text { if } k \geqslant 1, A_{3} \text { if } k \geqslant 1,-A_{4},-A_{5}, \\
-A_{6}, A_{7} \text { if } k \geqslant 1,-A_{7} \\
B_{4, k}, B_{4, k+1}, B_{5, k}, B_{5, k+1}, B_{6, k}, B_{6, k+1} \\
B_{7, k-1} \text { if } k \geqslant 2, B_{7, k+1}\end{array}$ \\
\hline$C_{1}$ & $(2,4,7,3,2)$ & $\begin{array}{c}-A_{2},-A_{3},-A_{4},-A_{5}, C_{3}, C_{4}, C_{7}, C_{8} \\
B_{2,1}, B_{2,2}, B_{4,1}, B_{5,1}, B_{7,0}, B_{7,1}\end{array}$ \\
\hline$C_{2}$ & $(2,5,7,3,2)$ & $\begin{array}{c}-A_{1},-A_{4}, C_{3}, C_{9} \\
B_{3,1}, B_{3,2}, B_{4,1}, B_{6,1}, B_{7,0}, B_{7,1}\end{array}$ \\
\hline$C_{3}$ & $(2,5,8,3,2)$ & $\begin{array}{c}A_{1}, A_{2},-A_{3},-A_{4}, C_{1}, C_{2}, C_{5}, C_{10} \\
B_{4,1}, B_{4,2}, B_{7,0}, B_{7,1}\end{array}$ \\
\hline$C_{4}$ & $(2,5,9,4,2)$ & $-A_{2}, A_{3}, C_{1}, C_{5}, B_{5,1}, B_{5,2}, B_{7,0}, B_{7,1}$ \\
\hline$C_{5}$ & $(2,6,10,4,2)$ & $A_{2}, A_{3}, C_{3}, C_{4}, B_{7,0}, B_{7,2}$ \\
\hline$C_{6}$ & $(3,5,7,3,2)$ & $-A_{1},-A_{2}, C_{7}, C_{9}, B_{1,2}, B_{2,1}, B_{2,2}, B_{3,1}, B_{3,2}, B_{4,1}$ \\
\hline$C_{7}$ & $(3,5,8,3,2)$ & $\begin{array}{l}A_{1},-A_{2}, A_{4}, C_{1}, C_{6}, C_{10} \\
\quad B_{2,1}, B_{2,2}, B_{4,1}, B_{4,2}\end{array}$ \\
\hline$C_{8}$ & $(3,5,9,4,2)$ & $A_{5}, C_{1}, B_{2,1}, B_{2,2}, B_{5,1}, B_{5,2}$ \\
\hline$C_{9}$ & $(3,6,8,3,2)$ & $\begin{array}{c}-A_{1}, A_{2}, A_{4}, C_{2}, C_{6}, C_{10} \\
B_{3,1}, B_{3,2}, B_{4,1}, B_{4,2}\end{array}$ \\
\hline$C_{10}$ & $(3,6,9,3,2)$ & $A_{1}, A_{2}, A_{4}, C_{3}, C_{7}, C_{9}, B_{4,1}, B_{4,2}$ \\
\hline
\end{tabular}




\section{Hille And M. Perling}

The rest of this section is devoted to the proof of Theorem 3.1.

\section{$3.1 c_{5}=3$}

Recall that the partial signature of the point $(-3,-1)$ is $\Sigma^{D}(-3,-1)=* * * *+++$. By the conditions (1), we immediately obtain the partial signature $*++++++$. So, the only way to prevent $H^{2}$ from showing up in the dual bundle is $\Sigma_{1}^{D}(-3,-1)=0$ (then, for the dual bundle, we have the signature $\left.\Sigma^{-D}(3,1)=0------\right)$. This in turn means that $c_{1}=2$. However, then, we have $\Sigma^{D}(-4,-2)=0++++0+$, and thus $\Sigma^{-D}(4,2)=0----0-$, hence we get $H^{1}$. We conclude that there are no divisors with $c_{5}=3$ and vanishing cohomology.

\section{$3.2 c_{5}=2$}

Here, conditions (1) read

$$
\begin{aligned}
& c_{1} \geqslant 1, \\
& c_{2} \geqslant 4, \\
& c_{3} \geqslant 7, \\
& c_{4} \geqslant 3 .
\end{aligned}
$$

We first consider $c_{4}=3$. Then $\Sigma(-3,0)=* * * 0++0$. If one of the $*$ is replaced by + , this implies that the dual signature will have at least two --intervals, independent on the other substitutions. So we obtain

$$
\begin{aligned}
& c_{1} \leqslant 3, \\
& c_{2} \leqslant 6, \\
& c_{3} \leqslant 9 .
\end{aligned}
$$

We treat these 27 possibilities case by case. First, let $c_{1}=1$. Then we have $\Sigma(-2,-1)=0 * *++0+$, and so we have $H^{1}$, leaving only 18 more cases. For these we write the following table.

\begin{tabular}{|c|c|c|c|c|}
\hline$c_{1}$ & $c_{2}$ & $c_{3}$ & $m$ & $\Sigma(m)$ \\
\hline 2 & 4 & 8 & $(-3,-2)$ & $+0+00-+$ \\
\hline 2 & 6 & - & $(-3,-1)$ & $0+* 0+++$ \\
\hline 2 & - & 9 & $(-3,-1)$ & $0 *+0+++$ \\
\hline 3 & 4 & - & $(-2,0)$ & $+0 *+++0$ \\
\hline 3 & 5 & 9 & $(-3,-1)$ & $+0+0+++$ \\
\hline 3 & 6 & 7 & $(-2,1)$ & $0+0+++-$ \\
\hline
\end{tabular}

This table contains a list of all values which have cohomology. For given values of $c_{1}, c_{2}, c_{3}$, the fourth columns contains a lattice point $m \in M$ with bad signature, which is displayed in the fifth column. Sometimes it suffices to display only a partial signature. Then the box for the corresponding $c_{i}$ contains a dash (-). All tuples which are not displayed in the above table represent cohomology free line bundles, namely the following.

\begin{tabular}{|c|c|c|c|c|}
\hline$c_{1}$ & $c_{2}$ & $c_{3}$ & $c_{4}$ & $c_{5}$ \\
\hline 2 & 4 & 7 & 3 & 2 \\
\hline 2 & 5 & 7 & 3 & 2 \\
\hline 2 & 5 & 8 & 3 & 2 \\
\hline 3 & 5 & 7 & 3 & 2 \\
\hline 3 & 5 & 8 & 3 & 2 \\
\hline 3 & 6 & 8 & 3 & 2 \\
\hline 3 & 6 & 9 & 3 & 2 \\
\hline
\end{tabular}




\section{A COUnTEREXAmple to King's CONJECture}

Now for $c_{4}=4$. We have $\Sigma(-3,-2)=* * *+0-+$ and thus we get the bounds

$$
\begin{aligned}
& c_{1} \geqslant 2, \\
& c_{2} \geqslant 5, \\
& c_{3} \geqslant 8 .
\end{aligned}
$$

Moreover, we have $\Sigma(-4,0)=* * * 0++0$ and, thus,

$$
\begin{aligned}
& c_{1} \leqslant 4, \\
& c_{2} \leqslant 8, \\
& c_{3} \leqslant 12 .
\end{aligned}
$$

Further, $\Sigma(-3,-1)=* * *++++$ and, hence,

$$
\begin{aligned}
& c_{1} \leqslant 2 \\
& c_{2} \leqslant 5 \text { or } \\
& c_{3} \leqslant 8 .
\end{aligned}
$$

We have $\Sigma(-4,-3)=* * * 0--+$ which implies

$$
c_{3} \geqslant 9
$$

and so the case $c_{3} \leqslant 8$ cannot occur. Also, the conditions imply that either $c_{1}=2$ or $c_{2}=5$, thus leaving 24 possibilities.

We first consider the case $c_{1}=2$. Then we have $\Sigma(-3,-2)=0 * * 000+$, which implies $c_{2} \leqslant 6$ and $c_{3} \leqslant 10$. Now we take $c_{2}=5$. Then $\Sigma(-4,-3)=+0 * 0--+$, so that we must have $c_{3}=9$. For $c_{2}=6$, we have $\Sigma(-4,-2)=00 * 000+$, which implies $c_{3}=10$. Indeed, we have found the following,

\begin{tabular}{|c|c|c|c|c|}
\hline$c_{1}$ & $c_{2}$ & $c_{3}$ & $c_{4}$ & $c_{5}$ \\
\hline 2 & 5 & 9 & 4 & 2 \\
\hline 2 & 6 & 10 & 4 & 2 \\
\hline
\end{tabular}

Now we consider $c_{2}=5$. We can assume that $c_{1} \geqslant 3$. Assume that $c_{3} \geqslant 10$. Then $\Sigma(-4,-3)=$ $+0+0--+$, so we have cohomology, hence $c_{3}=9$. For $c_{1}=4$, we have $\Sigma(-4,-3)=+0+0--+$ and thus cohomology, hence $c_{1}=3$, and indeed we have found the following.

\begin{tabular}{|c|c|c|c|c|}
\hline$c_{1}$ & $c_{2}$ & $c_{3}$ & $c_{4}$ & $c_{5}$ \\
\hline 3 & 5 & 9 & 4 & 2 \\
\hline
\end{tabular}

Now we go on with $c_{4} \geqslant 5$. Then we have $\Sigma(-4,-2)=* * *+00+$, which yields the conditions

$$
\begin{aligned}
& c_{1} \geqslant 4, \\
& c_{2} \geqslant 7, \\
& c_{3} \geqslant 11 .
\end{aligned}
$$

The signature $\Sigma(-3,-1)$ as before implies

$$
\begin{aligned}
& c_{1} \leqslant 2 \\
& c_{2} \leqslant 5 \text { or } \\
& c_{3} \leqslant 8 .
\end{aligned}
$$

Both conditions cannot be fulfilled simultaneously, and hence, for $c_{4} \geqslant 5$ there are no cohomologyfree bundles. 


\section{Hille And M. Perling}

\section{$3.3 c_{5}=1$}

Again, we start with the conditions (1), which read

$$
\begin{aligned}
& c_{1} \geqslant 0, \\
& c_{2} \geqslant 1, \\
& c_{3} \geqslant 2, \\
& c_{4} \geqslant 1 .
\end{aligned}
$$

Now we go for the different cases for $c_{1}$.

The case $c_{1}=0$. Here we have $\Sigma(-1,-1)=0 * * * 0-+$ so that all of the $*$ can only be substituted by zeros, and thus $c_{2}=1, c_{3}=2, c_{4}=1$ and indeed we have found

\begin{tabular}{|c|c|c|c|c|}
\hline$c_{1}$ & $c_{2}$ & $c_{3}$ & $c_{4}$ & $c_{5}$ \\
\hline 0 & 1 & 2 & 1 & 1 \\
\hline
\end{tabular}

with no other possibilities left.

The case $c_{1}=1$. We have $\Sigma(-2,-1)=0 * * * 00+$ which implies

$$
\begin{aligned}
& c_{2} \leqslant 3, \\
& c_{3} \leqslant 5, \\
& c_{4} \leqslant 2 .
\end{aligned}
$$

Let $c_{4}=1$, then $\Sigma(-1,0)=0 * * 0++0$, which implies

$$
\begin{aligned}
& c_{2} \leqslant 2, \\
& c_{3} \leqslant 3 .
\end{aligned}
$$

From these four cases, only $c_{2}=1, c_{3}=2$ has cohomology, as in this case $\Sigma(-1,-1)=+0+00-+$. We have found the following.

\begin{tabular}{|c|c|c|c|c|}
\hline$c_{1}$ & $c_{2}$ & $c_{3}$ & $c_{4}$ & $c_{5}$ \\
\hline 1 & 1 & 2 & 1 & 1 \\
\hline 1 & 2 & 2 & 1 & 1 \\
\hline 1 & 2 & 3 & 1 & 1 \\
\hline
\end{tabular}

Now let $c_{4}=2$. Then $\Sigma(-2,-1)=+* *+0-0$, so that

$$
\begin{aligned}
& c_{2} \geqslant 2, \\
& c_{3} \geqslant 3,
\end{aligned}
$$

leaving six cases. We write the following table, as before.

\begin{tabular}{|c|c|c|c|}
\hline$c_{2}$ & $c_{3}$ & $m$ & $\Sigma(m)$ \\
\hline 2 & 3 & $(-3,-2)$ & $+0-0--+$ \\
\hline 2 & 5 & $(-3,-2)$ & $+0+0--+$ \\
\hline 3 & 3 & $(-1,0)$ & $0+0+++0$ \\
\hline
\end{tabular}

Thus we have found the following.

\begin{tabular}{|c|c|c|c|c|}
\hline$c_{1}$ & $c_{2}$ & $c_{3}$ & $c_{4}$ & $c_{5}$ \\
\hline 1 & 2 & 4 & 2 & 1 \\
\hline 1 & 3 & 4 & 2 & 1 \\
\hline 1 & 3 & 5 & 2 & 1 \\
\hline
\end{tabular}




\section{A COUnterexample to King's CONJECture}

The case $c_{1} \geqslant 2$. Now for any $c_{1} \geqslant 2$, the point $\left(1-c_{1}, 0\right)$ has signature $\Sigma\left(1-c_{1}, 0\right)=+* * *++0$. So, we obtain general conditions

$$
\begin{aligned}
& c_{2} \geqslant 2 c_{1}-1, \\
& c_{3} \geqslant 3 c_{1}-2, \\
& c_{4} \geqslant c_{1} .
\end{aligned}
$$

We obtain another general condition as follows. Consider the signature $\Sigma\left(-c_{1}-1,0\right)=-* * *++0$. Assume that $c_{4} \geqslant c_{1}+2$. Then $\Sigma\left(-c_{1}, 0\right)=-+* *++0$ and so the $*$ can only be replaced by + , hence $c_{2} \geqslant 2 c_{1}+3$. The signature $\Sigma\left(-c_{2}-2,-1\right)$ then becomes either $-0 *-0+0$ or $-+*-0+0$ which both are bad. Thus, $c_{4}$ must be strictly smaller than $c_{1}+2$, and we have

$$
c_{4} \in\left\{c_{1}, c_{1}+1\right\} \text { for any value of } c_{1} \geqslant 2 \text {. }
$$

Now consider the signature $\Sigma\left(-c_{1}-1,-1\right)=0 * * * 0++$, which yields the following restrictions:

$$
\begin{aligned}
& c_{2} \leqslant 2 c_{1}+1, \\
& c_{3} \leqslant 3 c_{1}+2 .
\end{aligned}
$$

Now assume that $c_{4}=c_{1}$. From the signature $\Sigma\left(-c_{1}, 0\right)=0 * * 0++0$ we immediately obtain the conditions

$$
\begin{aligned}
& c_{2} \leqslant 2 c_{1}, \\
& c_{3} \leqslant 3 c_{1} .
\end{aligned}
$$

If $c_{2}=2 c_{1}-1$, we have the signature $\Sigma\left(-c_{1},-1\right)=+0 * 000+$, respectively $\Sigma(-2,-1)=+0 * 00++$, for the case $c_{1}=2$. In either case, we obtain

$$
c_{3} \leqslant 3 c_{1}-1 \text {. }
$$

For $c_{2}=2 c_{1}$, we have the signature $\Sigma\left(1-c_{1}, 1\right)=0+*+++-$, hence the $*$ cannot be replaced by - or 0 , thus we obtain $c_{3} \geqslant 2 c_{1}-1$. We cannot find any more restrictions and, in fact, we have found the following infinite series of cohomology-free line bundles.

\begin{tabular}{|c|c|c|c|c|}
\hline$c_{1}$ & $c_{2}$ & $c_{3}$ & $c_{4}$ & $c_{5}$ \\
\hline$k \geqslant 2$ & $2 k-1$ & $3 k-2$ & $k$ & 1 \\
\hline$k \geqslant 2$ & $2 k-1$ & $3 k-1$ & $k$ & 1 \\
\hline$k \geqslant 2$ & $2 k$ & $3 k-1$ & $k$ & 1 \\
\hline$k \geqslant 2$ & $2 k$ & $3 k$ & $k$ & 1 \\
\hline
\end{tabular}

Now let $c_{4}=c_{1}+1$. The signature $\Sigma\left(-c_{4},-1\right)=+* * 0--+$ yields

$$
\begin{aligned}
& c_{2} \geqslant 2 c_{1}, \\
& c_{3} \geqslant 3 c_{1}+1 .
\end{aligned}
$$

This leaves four possibilities of which we can only exclude the case $c_{2}=2 c_{1}, c_{2}=3 c_{1}+2$. Here we distinguish cases $c_{1}=2,3, \geqslant 4$. For $c_{1}=2$, we have $\Sigma(-3,-2)=+0+0--+$, for $c_{1}=3$ we have $\Sigma(-4,-2)=+0+00-+$ and for $c_{1} \geqslant 4$ we have $\Sigma\left(-c_{1}-1,-2\right)=+0+0+-+$, all of which are bad signatures. So, we have extracted three more series as follows.

\begin{tabular}{|c|c|c|c|c|}
\hline$c_{1}$ & $c_{2}$ & $c_{3}$ & $c_{4}$ & $c_{5}$ \\
\hline$k \geqslant 2$ & $2 k$ & $3 k+1$ & $k+1$ & 1 \\
\hline$k \geqslant 2$ & $2 k+1$ & $3 k+1$ & $k+1$ & 1 \\
\hline$k \geqslant 2$ & $2 k+1$ & $3 k+2$ & $k+1$ & 1 \\
\hline
\end{tabular}




\section{Hille And M. Perling}

\section{$3.4 c_{5}=0$}

We have the signatures $\Sigma(-1,0)=* * * * 0+0$ and $\Sigma(1,0)=* * * * 0-0$ which imply

$$
\begin{aligned}
& -1 \leqslant c_{1} \leqslant 1, \\
& -2 \leqslant c_{2} \leqslant 2, \\
& -3 \leqslant c_{3} \leqslant 3, \\
& -1 \leqslant c_{4} \leqslant 1 .
\end{aligned}
$$

As $c_{5}=0$, we can assume without loss of generality $c_{1} \geqslant 0$. We refine by case distinction by the values of $c_{1}$.

The case $c_{1}=0$. Here we can assume without loss of generality that $c_{4} \geqslant 0$. Let $c_{4}=0$ and thus without loss of generality $c_{2} \geqslant 0$. We have the following table.

\begin{tabular}{|c|c|c|c|}
\hline$c_{2}$ & $c_{3}$ & $m$ & $\Sigma(m)$ \\
\hline 0 & 2 & $(-1,-1)$ & $0-0---+$ \\
\hline 0 & 3 & $(-1,-1)$ & $0-+---+$ \\
\hline 1 & $\leqslant 0$ & $(1,0)$ & $-0-0++-$ \\
\hline 1 & $\geqslant 2$ & $(0,1)$ & $-0+0++-$ \\
\hline
\end{tabular}

Thus, we have found the following.

\begin{tabular}{|c|c|c|c|c|}
\hline$c_{1}$ & $c_{2}$ & $c_{3}$ & $c_{4}$ & $c_{5}$ \\
\hline 0 & 0 & 0 & 0 & 0 \\
\hline 0 & 0 & 1 & 0 & 0 \\
\hline 0 & 1 & 1 & 0 & 0 \\
\hline
\end{tabular}

Now let $c_{4}=1$. Then $\Sigma(-1,-1)=0 * * 0--+$ and hence $c_{2}=1$ and $c_{3}=2$. We have found the following.

\begin{tabular}{|c|c|c|c|c|}
\hline$c_{1}$ & $c_{2}$ & $c_{3}$ & $c_{4}$ & $c_{5}$ \\
\hline 0 & 1 & 2 & 1 & 0 \\
\hline
\end{tabular}

The case $c_{1}=1$. Assume first that $c_{4}=-1$. Then $\Sigma(0,1)=0 * *-++-$, which makes $c_{4}=-1$ impossible.

Now let $c_{4}=0$. We have $\Sigma(0,1)=0 * * 0++-$ which implies that $c_{2}=1$ and $c_{3}=1$. We have found the following.

\begin{tabular}{|c|c|c|c|c|}
\hline$c_{1}$ & $c_{2}$ & $c_{3}$ & $c_{4}$ & $c_{5}$ \\
\hline 1 & 1 & 1 & 0 & 0 \\
\hline
\end{tabular}

Finally, let $c_{4}=1$. Then $\Sigma(0,0)=+* *+000$, so

$$
\begin{aligned}
& c_{2} \geqslant 1, \\
& c_{3} \geqslant 1 .
\end{aligned}
$$

So we have reduced to six possibilities. Consider the following table.

\begin{tabular}{|c|c|c|c|}
\hline$c_{2}$ & $c_{3}$ & $m$ & $\Sigma(m)$ \\
\hline 1 & 1 & $(-1,-1)$ & $+0-0--+$ \\
\hline 1 & 3 & $(-1,-1)$ & $+0+0--+$ \\
\hline 2 & 1 & $(0,2)$ & $-0-+++-$ \\
\hline
\end{tabular}




\section{A COUnTEREXAmple to King's CONJECture}

The remaining cases are as follows, which finishes the classification.

\begin{tabular}{|c|c|c|c|c|}
\hline$c_{1}$ & $c_{2}$ & $c_{3}$ & $c_{4}$ & $c_{5}$ \\
\hline 1 & 1 & 2 & 1 & 0 \\
\hline 1 & 2 & 2 & 1 & 0 \\
\hline 1 & 2 & 3 & 1 & 0 \\
\hline
\end{tabular}

\section{Statement and proof of the theorem}

We represent the classification obtained in the previous section in Table 1 . We distinguish three types of line bundles, named by the letters $A$ to $C$, where the $B$-type bundles form infinite series. For a given cohomology-free bundle $\mathcal{L}$ the table shows the tuple $\left(c_{1}, c_{2}, c_{3}, c_{4}, c_{5}\right)$ and a list of all cohomology-free bundles $\mathcal{L}^{\prime}$ which have the property that $H^{k}\left(X, \mathcal{L}^{*} \otimes \mathcal{L}\right)=H^{k}\left(X, \mathcal{L} \otimes\left(\mathcal{L}^{\prime}\right)^{*}\right)=0$ for all $k>0$, which is a necessary condition for $\mathcal{L}$ and $\mathcal{L}^{\prime}$ for being part of the same strongly exceptional sequence. We say that $\mathcal{L}$ and $\mathcal{L}^{\prime}$ are compatible. Note that for the presentation purposes for the $B$-type bundles we have only listed the compatible bundles which are also of type $B$. For notation, $-A_{4}$ for instance means the line bundle $(-1,-1,-1,0,0)$. Now we state and prove our main result. Let $X$ be the toric surface as given in the introduction.

Theorem 4.1. On $X$ there are no strongly exceptional sequences of length 7 which consist of line bundles.

Proof. This is proved by inspection of the table and the exclusion principle. For example, assume that we have a strongly exceptional sequence of length 7 which contains $C_{10}$. Then the rest of the sequence can at most be selected from $A_{1}, A_{2}, A_{4}, C_{3}, C_{7}, C_{9}, B_{4,1}, B_{4,2}$. We see from the corresponding rows that at most one of the $A_{i}$ and at most one of the $C_{i}$ can be selected simultaneously. Hence, we can choose at most four elements from the list to complete the sequence. We conclude that a strongly exceptional sequence of length 7 which contains $C_{10}$ cannot exist. Thus, we can eliminate $C_{10}$ from the table.

As general rules we read off that at most two of the $A_{i}$ can be part of a strongly exceptional sequence, i.e. we have either $\pm A_{i}, i=1, \ldots, 7$ alone or $A_{i}, i=1, \ldots, 7$, and $A_{7}$ (respectively, $-A_{i}$ and $-A_{7}$ ) together, or $A_{i}$ and $-A_{7-i}, i=1, \ldots, 6$ (respectively, $-A_{i}$ and $A_{7-i}$ ) together.

Assume that a strongly exceptional sequence contains three bundles of type $B_{r, k}, B_{s, l}, B_{t, m}$. We read immediately off from the table that this is not possible if $r, s, t$ are pairwise distinct, hence at least two of the $r, s, t$ coincide. We also see that always $B_{r, k+1}-B_{r, k}=A_{7}$ for all $r$ and $B_{r, k+n}-B_{r, k}=n \cdot A_{7}$, so if two bundles of the same $B$-type are contained in a strongly exceptional sequence, these must be of the form $B_{r, k}, B_{r, k+1}$. Now given such a pair and assume that there exists one more $B_{s, l}$ together with this pair in a strongly exceptional sequence. Then $B_{r, k+1}-B_{s, l}=A_{i}$ for some $1 \leqslant i \leqslant 6$ and $B_{r, k}-B_{s, l}=-A_{7-i}$. If there exists another $B_{t, m}$ in this sequence, we have $B_{r, k+1}-B_{t, m}=A_{j}$ for $1 \leqslant i \leqslant 6$ and $B_{t, m}-B_{s, l}=A_{i}-A_{j}$, which is not possible. So we conclude that a strongly exceptional sequence can contain at most three of the $B$. This in turn, together with the above condition on the $A$, implies that a strongly exceptional sequence must contain at least one of the $C$.

We now proceed with $C_{9}$. We can only choose at most three of the compatible $B$ and at most one of the $A$. So we have to choose at least one out of $C_{2}$ and $C_{6}$. These two are mutually exclusive, so we can choose only one of them. Both choices restrict the choice of the $A$ to $-A_{1}$, which in turn is not compatible with $B_{4, k}$. So that we can choose at most two of the $B$, which is not enough, hence we can forget about $C_{9}$. 


\section{Hille And M. Perling}

For $C_{8}$, we can choose at most three of the $B$ and thus to obtain a strongly exceptional sequence, we have to choose both $A_{5}$ and $C_{1}$. However, $A_{5}$ is not compatible with $B_{2}$, so we cannot complete to a full sequence. Hence, we eliminate $C_{8}$.

For $C_{7}$, the bundles $C_{1}$ and $C_{6}$ are mutually exclusive, so in order to obtain an exceptional sequence of length seven, we have to choose one out of the $A$ and three out of the $B$. The $C$ leave only one choice for the $A$, namely $-A_{2}$, which in turn is not compatible with $B_{4, k}$, hence we can discard $C_{7}$.

For $C_{6}$ we have only the choice of at most one of the $A$ and of at most three of the $B$ left, which is not enough. So $C_{6}$ goes away.

For $C_{5}$, both pairs $C_{3}, C_{4}$ and $B_{1,2}, B_{7,2}$ are mutually exclusive, leaving not enough choices to complete the sequences.

Next we deal with $C_{4}$. The sequence must contain $C_{1}$ and $-A_{2}$, where the latter is not compatible with the $B_{7}$, so no $C_{4}$.

For $C_{3}$, we can choose at most one $A$ and at most one $C$. The $C$ are not compatible with $A_{1}$ and $A_{2}$, and $B_{4}$ and $B_{7}$ are not simultaneously compatible with one of $-A_{3}$ and $-A_{4}$, which does not leave enough choices to also choose $-A_{4}$, which is not compatible with $B_{4, k}$. So we can also exclude $C_{3}$.

In the remaining cases, for $C_{1}$ and $C_{2}$, we do not have any other $C$ at our disposal. Therefore, we cannot complete a sequence and so we can eliminate $C_{1}$ and $C_{2}$.

Altogether, we now have removed all of the $C$, and as we have seen above, it is not possible to complete to a strongly exceptional sequence of length 7 .

\section{REFERENCES}

AH99 K. Altmann and L. Hille, Strong exceptional sequences provided by quivers, Algebr. Represent. Theory 2 (1999), 1-17.

AKO04 D. Auroux, L. Katzarkov and D. Orlov, Mirror symmetry for weighted projective planes and their noncommutative deformations, Preprint (2004), math.AG/0404281.

Bei78 A. A. Beilinson, Coherent sheaves on $\mathbb{P}^{n}$ and problems of linear algebra, Funct. Anal. Appl. 12 (1978), 214-216.

BP05 A. Bergman and N. Proudfoot, Moduli spaces for Bondal quivers, Preprint (2005), math.AG/0512166.

Bon90 A. I. Bondal, Representation of associative algebras and coherent sheaves, Math. USSR Izv. 34 (1990), 23-42.

CM04 L. Costa and R. M. Miró-Roig, Tilting sheaves on toric varieties, Math. Z. 248 (2004), 849-865.

CS06 A. Craw and G. Smith, Projective toric varieties as fine moduli spaces of quiver representations, Preprint (2006), math.AG/0608183.

EMS00 D. Eisenbud, M. Mustata and M. Stillman, Cohomology on toric varieties and local cohomology with monomial supports, J. Symbolic Comput. 29 (2000), 583-600.

Ful93 W. Fulton, Introduction to toric varieties (Princeton University Press, Princeton, NJ, 1993).

Hil04 L. Hille, Exceptional sequences of line bundles on toric varieties, Mathematisches Institut Universität Göttingen Seminars WS03-04, ed. Y. Tschinkel (Universitätsverlag Göttingen, 2004), 175-190.

HKP05 M. Hering, A. Küronya and S. Payne, Asymptotic cohomological functions of toric divisors, Preprint (2005), math.AG/0501041.

Kaw05 Y. Kawamata, Derived categories of toric varieties, Preprint (2005), math.AG/0503102.

Kin97 A. King, Tilting bundles on some rational surfaces, unpublished manuscript (1997), http://www.maths.bath.ac.uk/ masadk/papers/tilt.ps. 


\section{A COUnTEREXAmple to King's CONJECTURE}

Oda88 T. Oda, Convex bodies and algebraic geometry, Ergebnisse der Mathematik und ihrer Grenzgebiete (3), vol. 15 (Springer, Berlin, 1988).

Rud90 A. N. Rudakov, Helices and vector bundles: seminaire Rudakov, London Mathematical Society Lecture Note Series, vol. 148 (Cambridge University Press, Cambridge, 1990).

Lutz Hille hille@math.uni-bielefeld.de

Fakultät für Mathematik, Universität Bielefeld, Postfach 100131, 33501 Bielefeld, Germany

Markus Perling perling@mozart.ujf-grenoble.fr

Institut Fourier — UMR5582, 100 rue des Maths, BP 74, 38402 St. Martin d'Heres, France 